\title{
Reference Undulator Measurement Results
}

\author{
Zachary Wolf, Yurii Levashov \\ SLAC
}

August 26, 2009

\begin{abstract}
The LCLS reference undulator has been measured 22 times during the course of undulator tuning. These measurements provide estimates of various statistical errors. This note gives a summary of the reference undulator measurements and it provides estimates of the undulator tuning errors.
\end{abstract}

\section{$1 \quad$ Introduction $^{1}$}

The LCLS reference undulator (SN13) has been measured 22 times over a period of 2 years. The purpose of the reference was to make sure the measurement systems were stable during the tuning of all the undulators. The large number of measurements also allows us to make error estimates of the tuning parameters. This paper presents a summary of the reference undulator measurements and it provides estimates of how well the undulators are tuned.

In this note tolerances on the tuning parameters are quoted. All tolerances come either from an LCLS requirements document ${ }^{2}$ or from a parameter database. ${ }^{3}$

Not all of the 22 data sets are used. Data set 1 was an early measurement before production measurements started. Some refinements to the measurement systems were still being done. Data set 16 was a quick check of the reference undulator after a temperature excursion in our lab. No mechanical measurements were done. Data set 20 was taken as a check after software upgrades. Data sets 1, 16, and 20 are all excluded from the analyses in this note.

The undulator tuning was divided into two runs. This will be indicated below on several plots. After most of the undulators were tuned in the first production run, it was found that essentially all were damaged due to temperature excursions during storage. Furthermore, it was discovered that the beam pipe was designed with interferences with the tuning shims. A second production run was required to fix these problems.

\section{Mechanical Measurements}

The reference undulator was fiducialized for each data set. The fiducialization results will be presented in a later section. As part of the process, however, a number of mechanical measurements were made with the CMM. We present some of these measurements here to give information about the undulator and to show the repeatability of the mechanical measurements.

\footnotetext{
${ }^{1}$ Work supported in part by the DOE Contract DE-AC02-76SF00515. This work was performed in support of the LCLS project at SLAC.

${ }^{2}$ H. D. Nuhn, et. al., "General Undulator System Requirements", LCLS Physics Requirements Document \#1.4-001, rev. 4., April, 2008.

${ }^{3}$ The parameters are on the web at http://www-ssrl.slac.stanford.edu/lcls/parameters.html.
} 
Figure 1 shows the y-position of the center of the gap with respect to the fiducialized beam axis for each of the 226 pole pairs in the undulator and for each data set. Ideally, the plot would be a straight line at 0 . Note that the undulator is not particularly straight, especially the exit end. This is fairly typical. For extreme cases, we used the wedge shims to straighten the gap. This was not done for the reference undulator.

Figure 2 shows the $\mathrm{x}$-position of the edges of the poles with respect to the fiducialized beam axis. Many measurement errors affect the $\mathrm{x}$-position of the beam axis and thus change the x-position shown in the plot. This overall offset error will be discussed at length in a later section. There is no provision built into the undulator to correct the x-position of individual poles, and thus straighten the profiles in the plot.

Figure 3 shows the cant angle of each pole. There is considerable variation from pole to pole, however, there is no means to correct the cant angle built into the undulator. The nominal cant angle is $4.5 \mathrm{mrad}$.

Figure 4 shows the gap height at the beam axis position. Again, there is considerable variation from pole to pole. The nominal gap height is $6.8 \mathrm{~mm}$. There is no means to correct the gap height of an individual pole pair built into the undulator. The tapered shims, however, are used to correct the average gap height over many pole pairs (approximately 10). This is important for setting the $K$ value and making phase corrections.

Figure 5 shows the gap height at the beam axis position relative to the first data set. (The "first data set" is the first data set in the plot.) The gap is consistently larger for data sets after the first. In addition, the ends of the undulator seem to have moved relative to the first data set.

\section{Trajectories, Phase, Etc.}

The electron trajectories, phase, etc. are calculated from the measured magnetic field. The magnetic field in the undulator is measured with a hall probe transported down the undulator gap along a straight line determined by a precision granite bench. The bench is aligned to be straight within \pm 5 $\mu \mathrm{m}$ in $y$ and $\pm 10 \mu \mathrm{m}$ in $x$. Repeatability of the magnetic measurements is essential for repeatability of the calculated quantities between data sets. Repeatability also allows us to use single scans to do the measurements instead of averaging multiple scans.

The tolerance on the trajectory straightness requires that the maximum trajectory walkoff per $10 \mathrm{~m}$ is $5 \mu \mathrm{m}$, the maximum first field integral in the undulator is $40 \mu \mathrm{Tm}$, and the maximum second field integral is $50 \mu \mathrm{Tm}^{2}$. We take the maximum trajectory walkoff within an undulator to be 2 $\mu \mathrm{m}$. Using a beam energy $E=13.64 \mathrm{GeV}$, the first integral specification requires the exit angle of the beam to be less than $8.8 \times 10^{-7} \mathrm{rad}$. The second field integral specification requires the exit position of the beam to be less than $1.1 \mu \mathrm{m}$ from the entrance position.

The tolerance on the phase of the electron motion relative to the radiation wave requires that they be synchronized to 10 degrees. This applies to all points in the undulator. In addition, the phase change in a cell is specified. A cell is defined as a length of $3.656 \mathrm{~m}$ centered on the undulator. The phase change in a cell must be $113 \times 360$ degrees within \pm 10 degrees.

In order to investigate the repeatability of the measurements, consider the changes in the measured peak fields. Figure 6 shows the peak field at every pole for the data sets, minus the peak field from the first data set. The figure shows some random noise in the measurements at the level of one gauss, or so. The noise gets larger toward the exit end of the undulator. We believe this is due to an effect we were told about by the hall probe representative, namely that the hall probe warms up as it passes through the alternating magnetic field. These errors will cause some trajectory and phase scatter, as we will see. The general shape of the peak field changes agrees with the gap height changes shown in figure 5.

Figure 7 shows the calculated $\mathrm{x}$-trajectory for a $13.64 \mathrm{GeV}$ electron going through the undulator for each of the data sets. The results overlap well. Note, however, that a correction is made to 
the hall probe measurements. The NMR calibrations of the hall probe can not take out zero offsets at the level required for these measurements. A small offset correction, typically around $0.2 \mathrm{G}$, is added to the measurements to make the field integrals agree with the long coil measurements. The difference in the trajectories is largely due to errors in the offset correction. A discussion of the long coil measurements follows, and the errors will be discussed again.

Figure 8 shows the y-trajectories for the data sets. Again, the differences are largely due to errors in the offset corrections coming from the long coil measurements.

Figure 9 shows the phase of the electron motion relative to the phase measured in the first data set. Changes are typically less than $2 \mathrm{deg}$. This phase is calculated using the wavelength the undulator is radiating at. It isolates phase and does not show the effect of errors in the $K$ value.

Figure 10 shows the phase of the electron motion relative to the nominal light wave of $\lambda=1.5 \AA$ and relative to the phase measured in the first data set. Again, changes are typically less than 2 deg. Errors in the $K$ value are included in this plot.

Figure 11 shows the phase change relative to the nominal light wave in a cell relative to the first data set. Changes among the data sets are below 4 deg.

\section{$4 \quad$ Field Integrals}

The tolerance specification on the undulator field integrals requires that the first field integral be less than $40 \mu \mathrm{Tm}$, and the second field integral be less than $50 \mu \mathrm{Tm}^{2}$. This applies to both $B_{x}$ and $B_{y}$.

The first and second field integrals of $B_{x}$ and $B_{y}$ are measured with a long coil. ${ }^{4}$ The accuracy of the system was checked ${ }^{5}$ and determined to be $\pm 5 \mu \mathrm{Tm}$ for the first integrals and $\pm 10 \mu \mathrm{Tm}^{2}$ for the second integrals. We found, however, that the measurements in the reference undulator drifted over time, as will be shown below.

Figure 12 shows the first and second field integrals of $B_{x}$ and $B_{y}$ over a $\pm 6 \mathrm{~mm}$ range in the $\mathrm{x}$-direction. The reference undulator, being one of the first undulators measured, was not tuned to meet the requirements over the full $\pm 6 \mathrm{~mm}$ range, only in the center. Note that there is a spread in the measurements. We believe this is due to the coil straightness changing. The coil was initially adjusted to be straight to $\pm 100 \mu \mathrm{m}$. It was not routinely checked, however, until January, 2008. After that, the coil was straightened before each measurement.

Figure 13 shows the field integrals at the $x=0$ position as a function of time. After the routine coil straightening, the measurements appear more repeatable. We believe the problem is that a number of our shims ( $B_{x}$ shims, quadrupole and sextupole correction shims) have field gradients. If the coil is at a different position in the gradient of the shim, the field integral will change. In spite of this, the rms variation of the first integral of $B_{x}$ at the $x=0$ position is $9.98 \mu \mathrm{Tm}$, and the rms variation of the second integral of $B_{x}$ is $17.4 \mu \mathrm{Tm}^{2}$. The rms variation of the first integral of $B_{y}$ at the $x=0$ position is $6.65 \mu \mathrm{Tm}$, and the rms variation of the second integral of $B_{y}$ is $12.3 \mu \mathrm{Tm}^{2}$. These values are well below the tolerance limit and close to the measurement accuracy.

As noted above, the field integral measurements from the long coil are used to correct offsets in the hall probe measurements. The changes in the field integral measurements are largely responsible for the changes in the trajectories.

\section{Beam Axis Position}

The tolerance on setting the $K$ value of an undulator is $\Delta K / K<1.5 \times 10^{-4}$. With the canted poles, the $K$ value changes with with the x-position of the beam axis. The $\cosh (y)$ dependence

\footnotetext{
${ }^{4}$ Z. Wolf, "Undulator Field Integral Measurements", LCLS-TN-05-22, August, 2005.

${ }^{5}$ Z. Wolf, Y. Levashov, "Undulator Long Coil Measurement System Tests", LCLS-TN-07-3, April, 2007.
} 
of the field makes the $K$ value also change with the y-position of the beam axis. Each time the reference undulator was measured, the position of the beam axis was determined relative to fixed tooling balls on the undulator. The repeatability of the beam axis position allows an estimate of the error in the relative $K$ value between undulators during production measurements.

The beam axis position determined during the fiducialization process is sensitive to many errors. Figure 14 illustrates various errors which can occur. Starting in the upper left corner, if the temperature in the lab is not correct, the permanent magnets in the undulator will not have the proper strength. The axis position in the canted poles will change in order to get to the specified $\mathrm{K}$ value. A complication, however, is that the hall probe output also changes. A combination of magnet strength change and hall probe measurement change leads to a change in the axis position.

The upper middle figure illustrates the fact that the hall probe can not be placed exactly on the ideal beam axis. It is always close, but a small error is present.

The upper right figure illustrates the possibility of an error happening during the fiducialization. For instance, a fiducialization magnet can be bumped while the undulator is being moved from the measurement bench to the CMM. There are also errors in the CMM measurements and hall probe measurements which cause errors in the fiducialization.

The center left figure illustrates that hall probe calibration errors and hall probe rotation errors cause errors in the field measurements. These in turn are compensated by a shift in axis position so the measurements, with their errors, give the specified $\mathrm{K}$ value. The fiducialized beam axis thus has an error.

The center middle figure shows that the $B_{x}$ and $B_{y}$ hall elements are at different vertical positions. Only one of the two probes can be on the midplane during a $K$ measurement. If the $B_{y}$ probe is off the midplane, the $\cosh (y)$ field dependence causes an error. If the $B_{x}$ probe is off the midplane, the planar hall effect from the $B_{z}$ field causes an error. For $K$ measurements, the $B_{y}$ probe is on the midplane and the $B_{x}$ field is corrected using the long coil measurement. In general, the hall elements are close enough together (around $20 \mu \mathrm{m}$ vertical displacement) and the corrections using the long coil are good enough that this problem is manageable.

The center right figure shows that the planar hall effect causes trajectory curvature, which causes errors in the $\mathrm{K}$ value. This effect is minimized by using the long coil to correct the hall probe measurements. Undulator straightness deviation contributes to this error.

It is also possible that the reference undulator physically changes, causing changes in the fiducialized beam axis position. The lower left figure illustrates the fact that temperature excursions permanently change the undulators. ${ }^{6}$ This can cause the beam axis position to change.

Undulator changes are also possible due to stretching of the bolts which hold the pole assemblies. We have had problems with bolts developing an hourglass shape due to the large forces. If the bolts stretch, the gap is reduced and the field in the gap changes. The beam axis will shift in the canted poles to compensate.

A few of the errors discussed are measurable. Figure 15 shows that the undulator was always fiducialized to the same $K$ value to within $1.5 \times 10^{-5}$. Figure 16 shows the temperature of the reference undulator for all the data sets. It was always measured within $0.1 \mathrm{C}$ of $20 \mathrm{C}$. Corrections for temperature differences are possible in theory, but the exact strength of the undulator as a function of temperature is unknown (it is presently being studied) and the effect of temperature on the hall probe is unknown. Most of the other errors discussed above are not quantifiable, or can not be measured with sufficient accuracy.

Figure 17 shows the x-position of the fiducialized beam axis for each data set compared to the first data set. The x-position is determined relative to fixed tooling balls on the undulator. Using the nominal $4.5 \mathrm{mrad}$ cant angle, if the x-position changes by $195 \mu \mathrm{m}$, the $K$ value changes by $\Delta K / K=1.5 \times 10^{-4}$. In the first production run, the rms deviation of the measurements was 60 $\mu \mathrm{m}$. Using the nominal cant angle, this corresponds to an rms fractional variation in the $K$ value of

\footnotetext{
${ }^{6}$ Z. Wolf, Y. Levashov, E. Reese, "Undulator Changes Due To Temperature Excursions", LCLS-TN-08-8, September, 2008 .
} 
$\Delta K / K=4.6 \times 10^{-5}$. In the second production run, the rms deviation of the beam axis x-position was $35 \mu \mathrm{m}$. This corresponds to an rms $K$ variation of $\Delta K / K=2.7 \times 10^{-5}$. The repeated measurements of the reference undulator give a good indication that the relative $K$ value between undulators was set with $\Delta K / K \simeq 2.7 \times 10^{-5}$ during the production tuning.

Figure 18 shows the y-position of the fiducialized beam axis for each data set. If the y-position changes by $83 \mu \mathrm{m}$, the $K$ value changes by $\Delta K / K=1.5 \times 10^{-4}$ due to the $\cosh (y)$ field dependence. For the first production run, the rms variation of the fiducialized beam axis y-positions was $9 \mu \mathrm{m}$, and for the second production run it was $4 \mu \mathrm{m}$. Note that these values are far below the $83 \mu \mathrm{m}$ tolerance.

One may wonder whether the reference undulator changed with time due to temperature excursions in the measurement lab. Figure 19 shows the history of the lab air temperature. Note that the air conditioner failed several times during the measurements. In particular, there were three times that the temperature rose above $22 \mathrm{C}$ during the production measurements. These events are indicated on figure 20, showing that these temperature excursions do not appear to be responsible for the changes in the beam axis x-position. This is likely due to the short duration of the temperature excursions and the large thermal capacity of the undulator.

Many calibrations of the hall probe were done during the production runs. They are indicated in figure 21. After each calibration, the hall probe required alignment, which potentially introduced an error. Because of the number of hall probe calibrations and alignments, and their random errors, it seems unlikely that calibration related errors are responsible for the systematic changes in the beam axis x-position. These errors probably do contribute, however, to the random scatter in the plot.

If the temperature sensors used at the measurement bench drifted with time, the $K$ values would be in error. $K$ is set to a fixed number when the measured temperature is $20 \mathrm{C}$. The temperature dependence of the undulator would cause an error in the field if the actual temperature was different than the measured temperature. The temperature measurements were checked several times during the production measurements. In April, 2009, we received a new reference thermometer. We compared the temperature sensor readings with the reference thermometer and obtained the results in the following table. Essentially no drift in the sensor measurements occurred over the two years of undulator tuning.

\begin{tabular}{|c|c|c|c|}
\hline Sensor \# & Sensor Meas $(\mathrm{C})$ & Reference $(\mathrm{C})$ & Difference $(\mathrm{C})$ \\
\hline 1 & 19.96 & 19.958 & .002 \\
\hline 2 & 19.97 & 19.966 & .004 \\
\hline 3 & 19.96 & 19.956 & .004 \\
\hline 4 & 19.94 & 19.943 & -.003 \\
\hline 5 & 19.93 & 19.931 & -.001 \\
\hline 6 & 19.88 & 19.875 & .005 \\
\hline
\end{tabular}

\section{Reference Dipole}

We placed a small, $3.7 \mathrm{kG}$ permanent magnet dipole on the measurement bench. It was carefully built so that an NMR probe works in it. Part of our procedure for each data set is to measure the reference dipole both with the hall probe and with an NMR probe. The field in the dipole is not perfectly uniform and we are not sure that the hall probe measurements and NMR measurements are done at the same location. In addition, the large sample in the NMR probe averages over a volume of the field. The locations of the measurements, however, are done in a very repeatable manner, so that relative changes in the hall probe reading compared to the NMR would indicate a problem. Figure 22 shows how the relative difference in the measurements changed with time. Note that changes are less than $1.0 \times 10^{-4}$. This shows that our hall probe calibrations were stable 
and that the probe was replaced on the bench without significant rotation errors during the time of the measurements.

\section{Conclusion}

We measured the reference undulator many times during the tuning of the LCLS undulators. These data sets give estimates of the random errors in the tuned undulators. The measured trajectories in the reference undulator are stable and straight to within $\pm 2 \mu \mathrm{m}$. Changes in the phase errors are less than $\pm 2 \mathrm{deg}$ between data sets. The phase advance in the cell varies by less than $\pm 2 \mathrm{deg}$ between data sets. The rms variation between data sets of the first integral of $B_{x}$ is $9.98 \mu \mathrm{Tm}$, and the rms variation of the second integral of $B_{x}$ is $17.4 \mu \mathrm{Tm}^{2}$. The rms variation of the first integral of $B_{y}$ is $6.65 \mu \mathrm{Tm}$, and the rms variation of the second integral of $B_{y}$ is $12.3 \mu \mathrm{Tm}^{2}$. The rms variation of the x-position of the fiducialized beam axis is $35 \mu \mathrm{m}$ in the final production run This corresponds to an rms uncertainty in the $\mathrm{K}$ value of $\Delta K / K=2.7 \times 10^{-5}$. The rms variation of the y-position of the fiducialized beam axis is $4 \mu \mathrm{m}$ in the final production run. 


\section{List Of Figures}

1. $y$-position of the center of the undulator gap at each pole. $y=0$ is the fiducialized beam axis.

2. $\mathrm{x}$-position of the edge of the poles. $\mathrm{x}=0$ is the fiducialized beam axis.

3. Cant angle of each pole.

4. Gap height at each pole at the fiducialized beam axis.

5. Gap height at each pole at the fiducialized beam axis relative to the first data set.

6. Fitted peak field for each pole relative to the first data set in the figure.

7. x-trajectory for each data set.

8. y-trajectory for each data set.

9. Phase between the electron beam and the light emitted by the undulator relative to the first data set.

10. Phase relative to the nominal radiation wave at $1.5 \AA$, relative to the first data set.

11. Phase relative to the nominal radiation wave in the cell, relative to the first data set.

12. Field integrals vs $\mathrm{x}$-position.

13. Field integrals at $x=0$ as a function of time.

14. Illustration of the errors which cause a change in the fiducialized beam axis position.

15. $K$ value that the undulator was fiducialized to relative to the first data set.

16. Undulator temperature for each data set.

17. x-position of the fiducialized beam axis relative to the first data set.

18. y-position of the fiducialized beam axis relative to the first data set.

19. Air temperature in the MMF during the production measurements.

20. $\mathrm{x}$-position of the fiducialized beam axis with the temperature excursions over $22 \mathrm{C}$ indicated.

21. x-position of the fiducialized beam axis with the hall probe calibrations indicated.

22. Change in the comparison of the hall probe to the NMR probe in the reference dipole. 

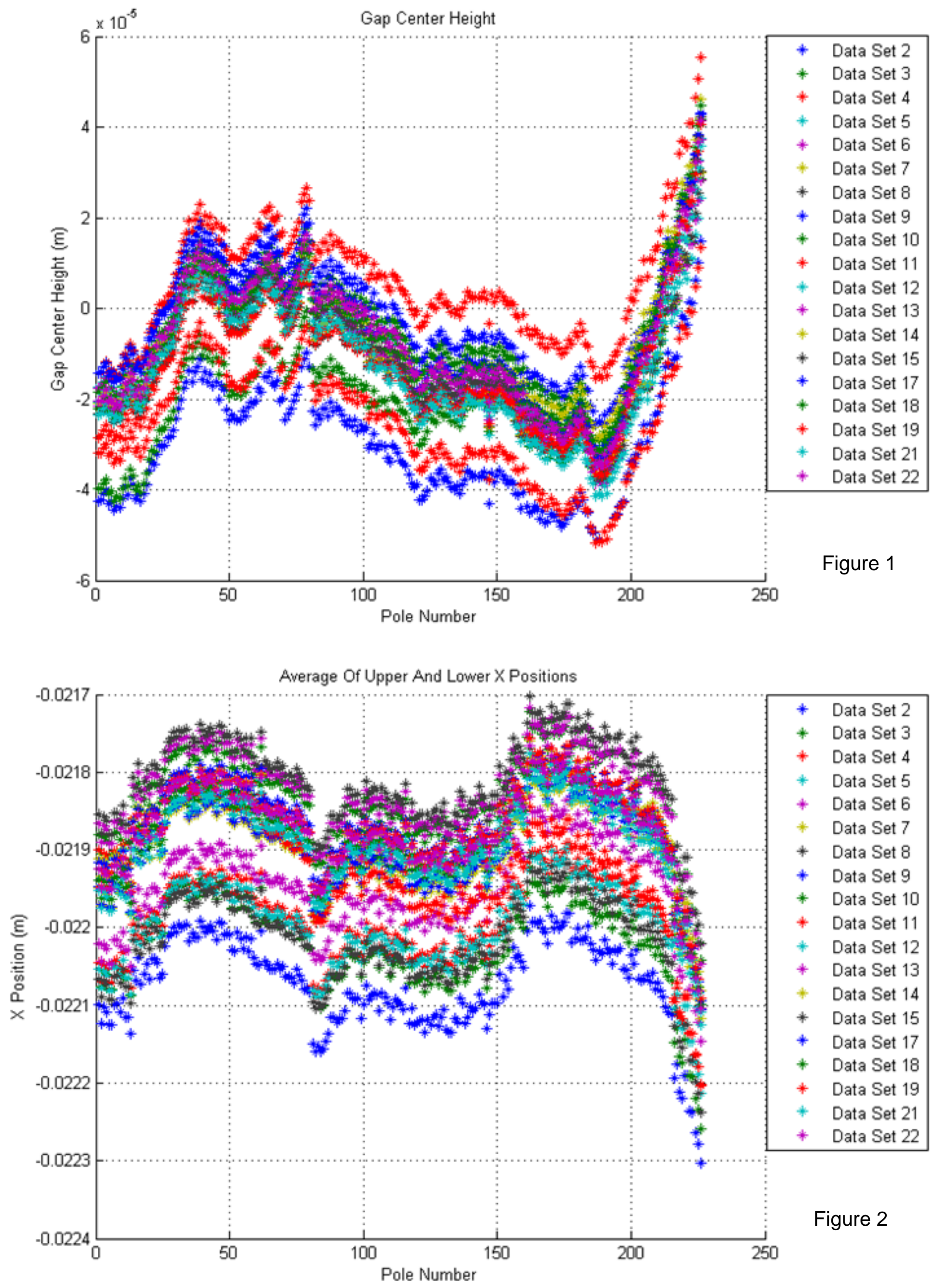

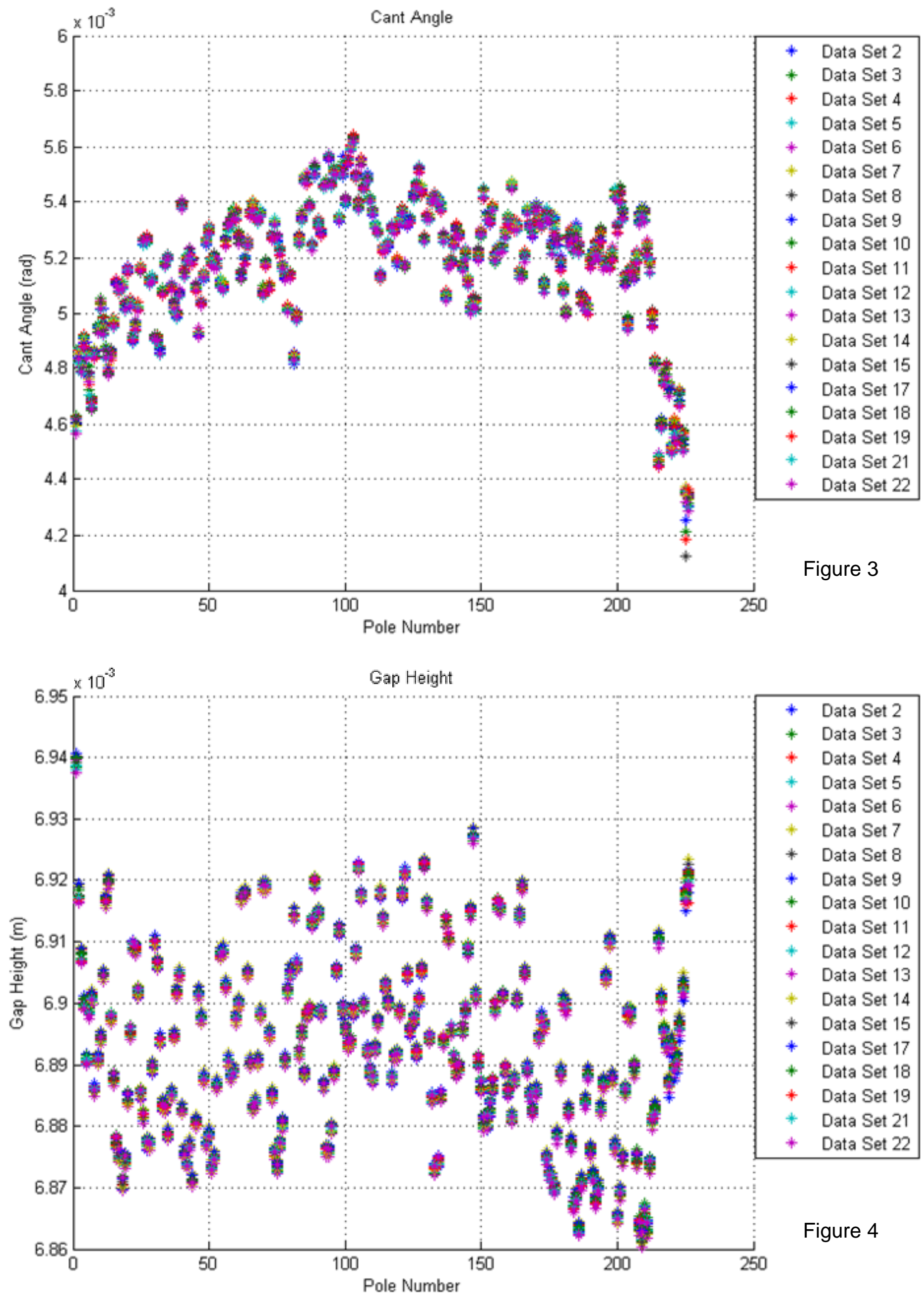

Figure 4 

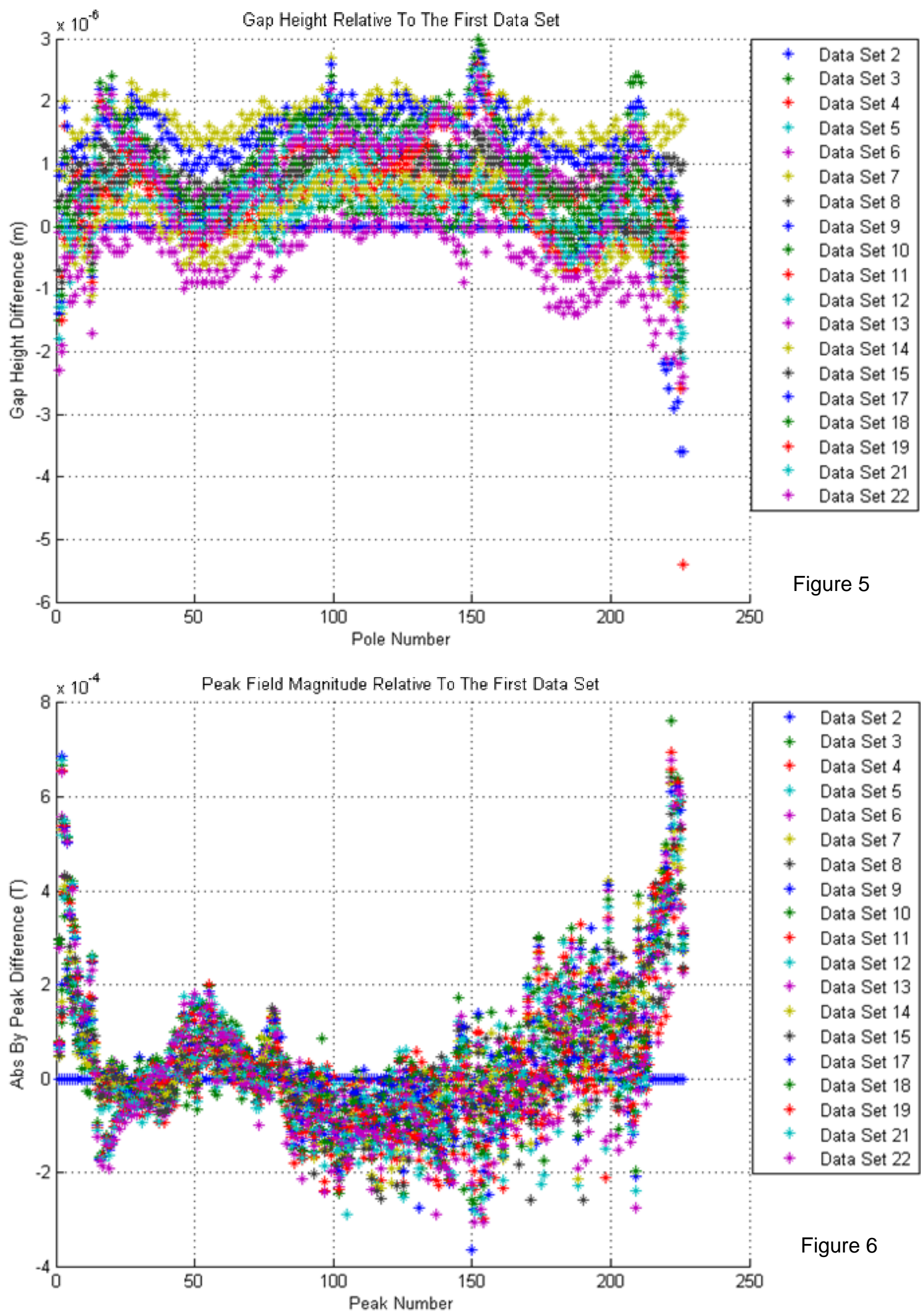

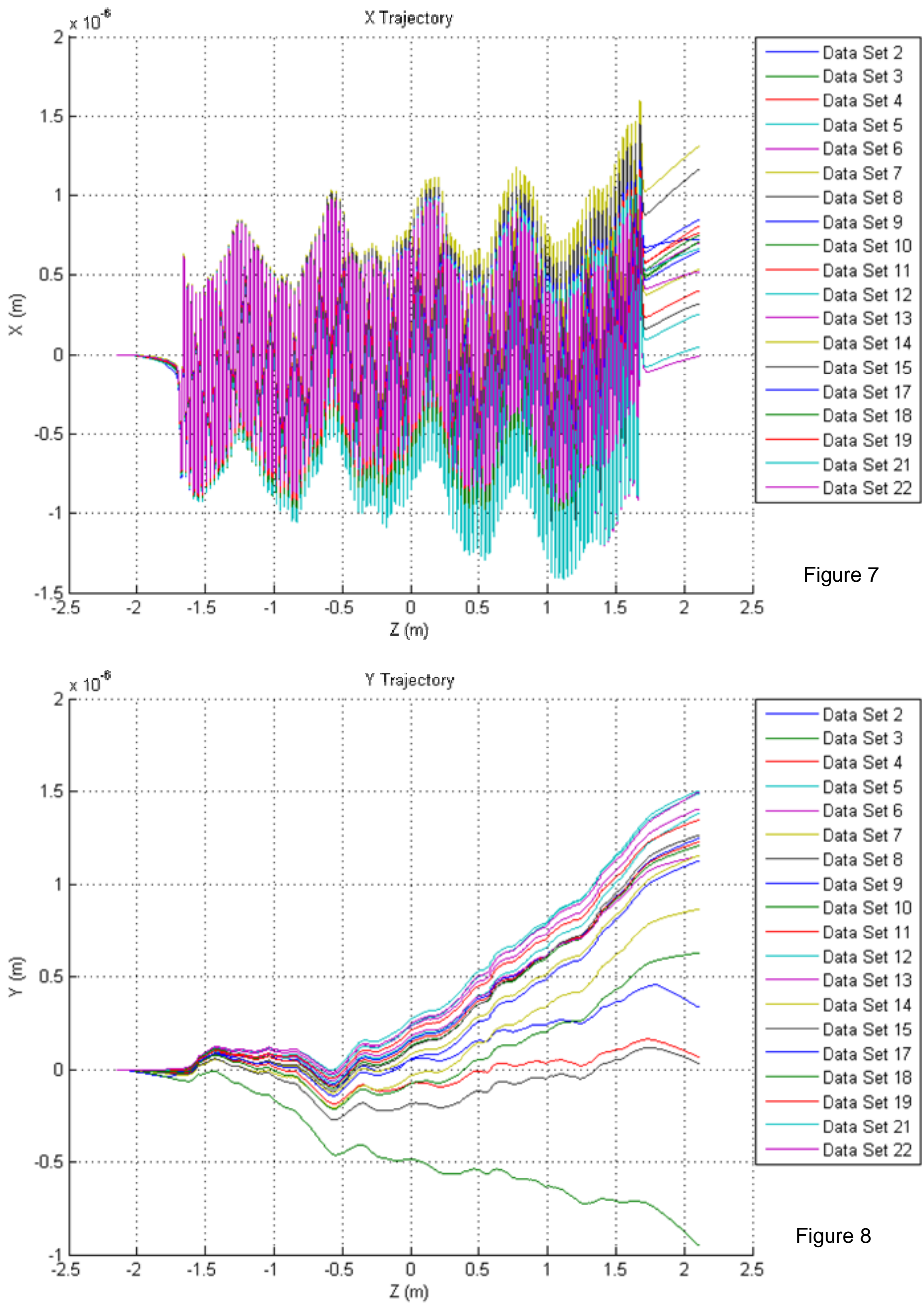

Figure 8 
Phase Relative To The Phase Of The First Data Set

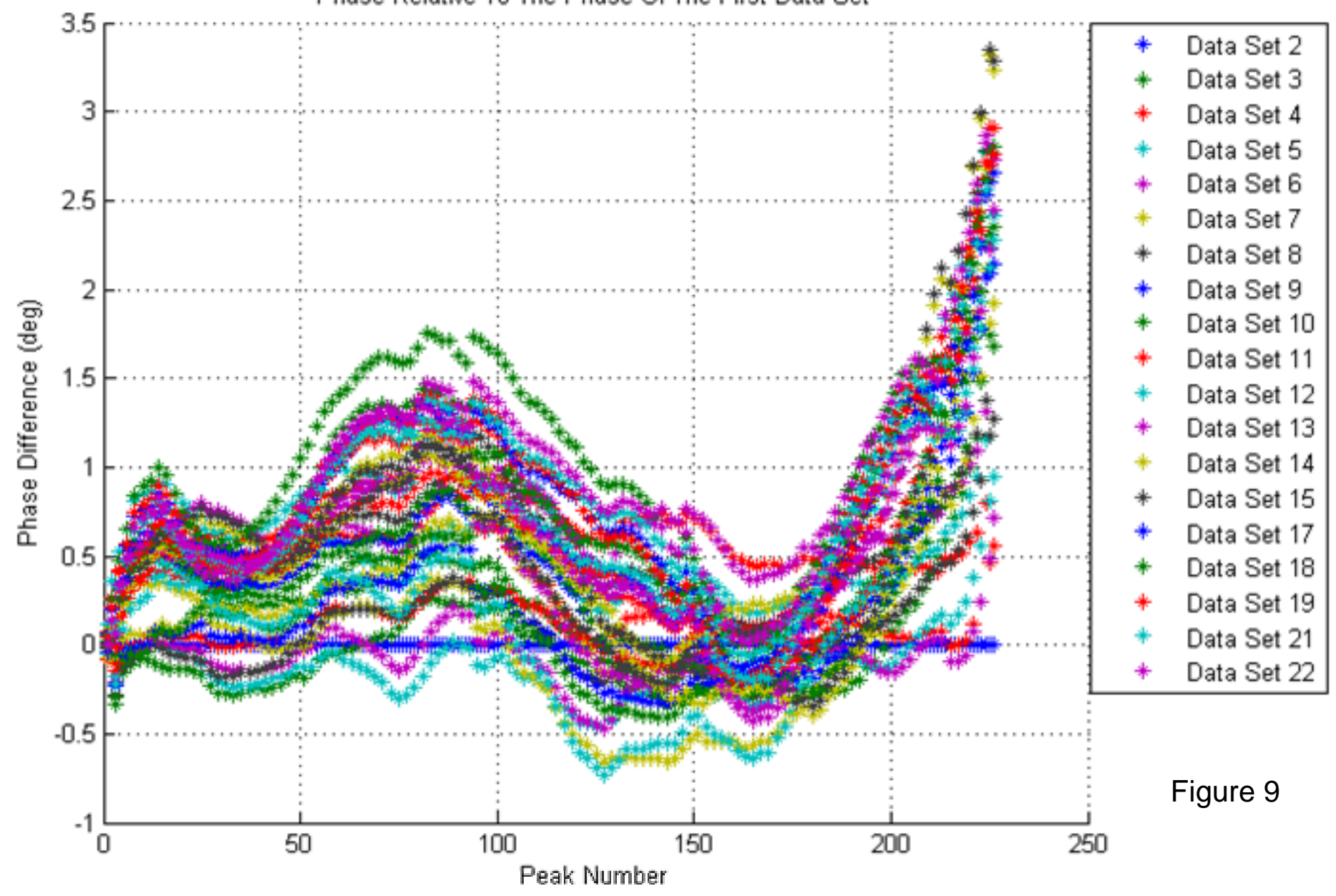

Phase Relative To The Nominal Wave, Relative To The Phase Of The First Data Set

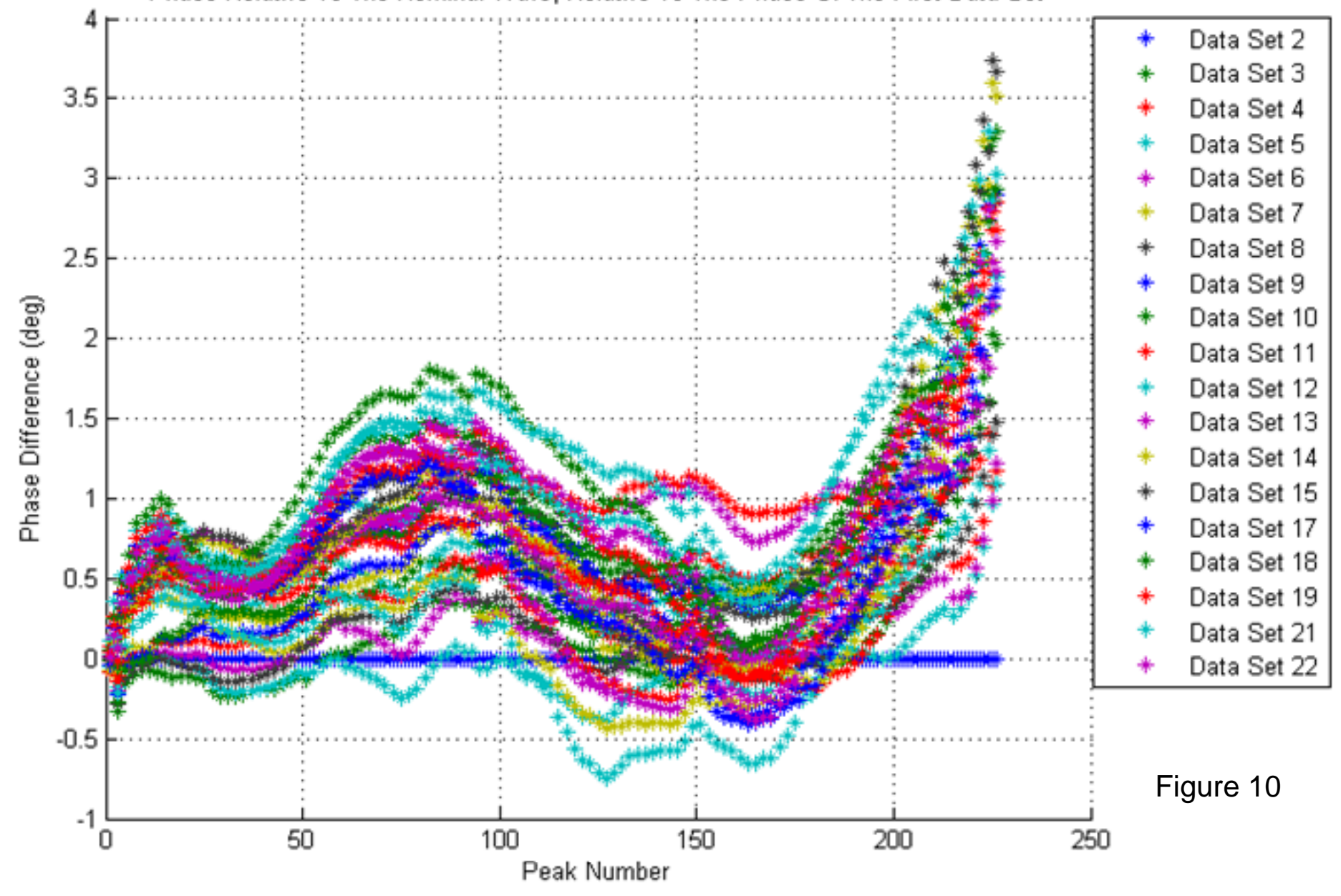


Change In Cell Phase Relative To The Nominal Wave Compared To The First Data Set

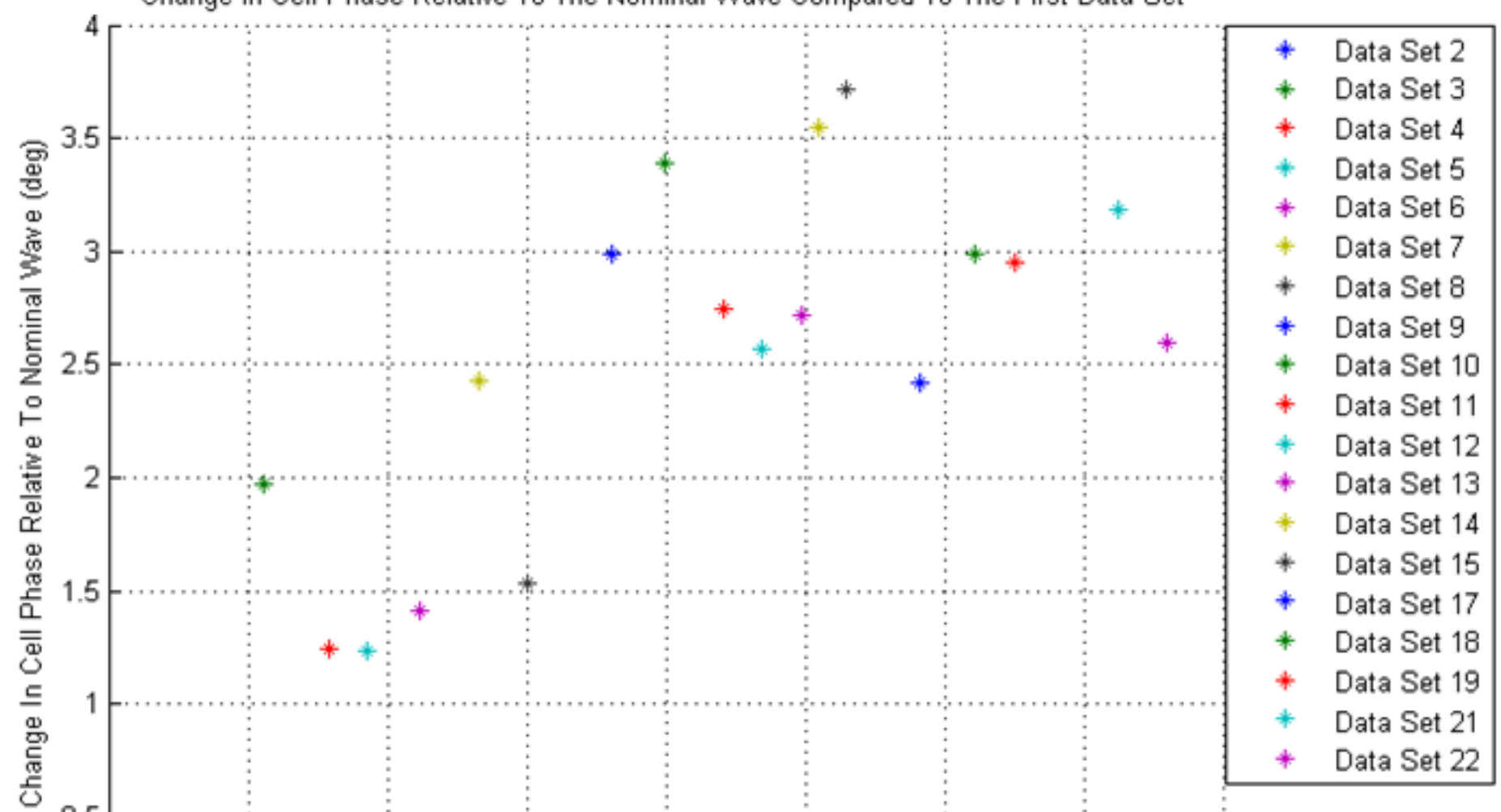

Figure 11
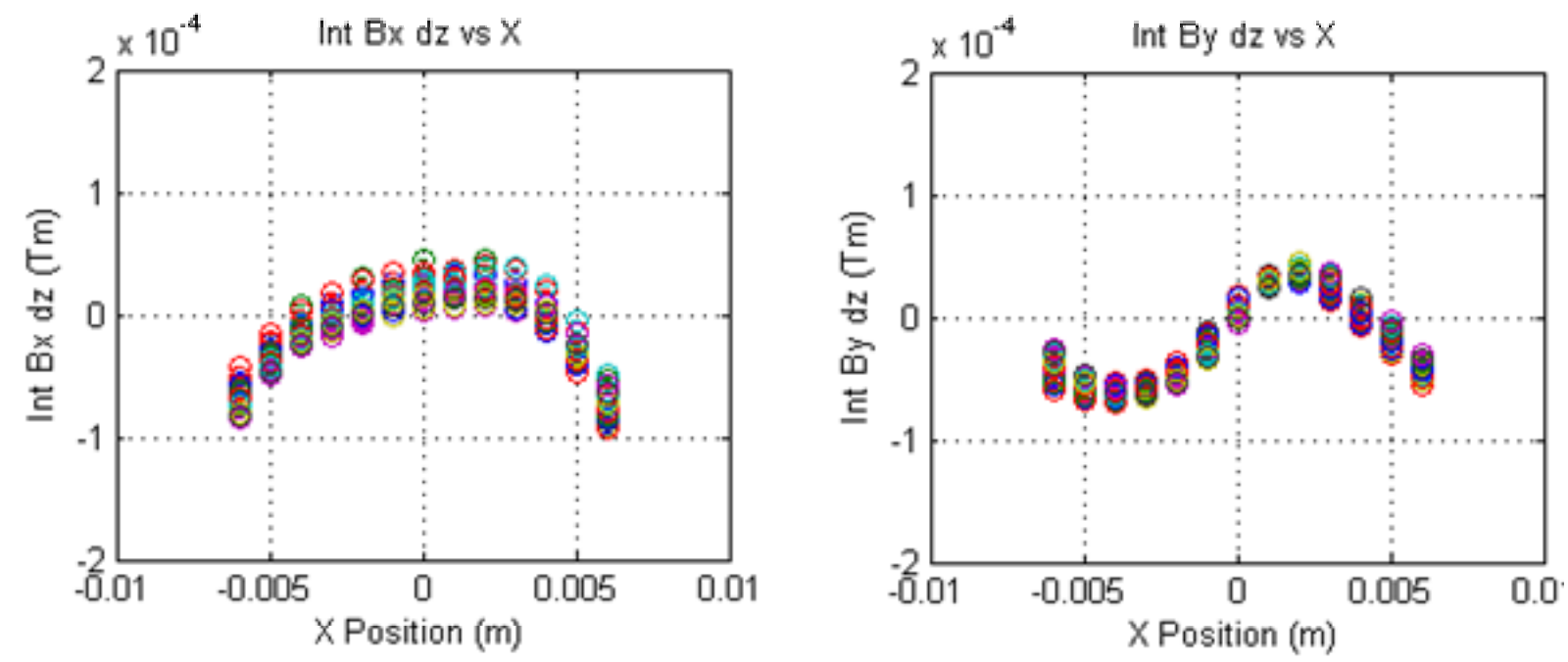

Figure 12
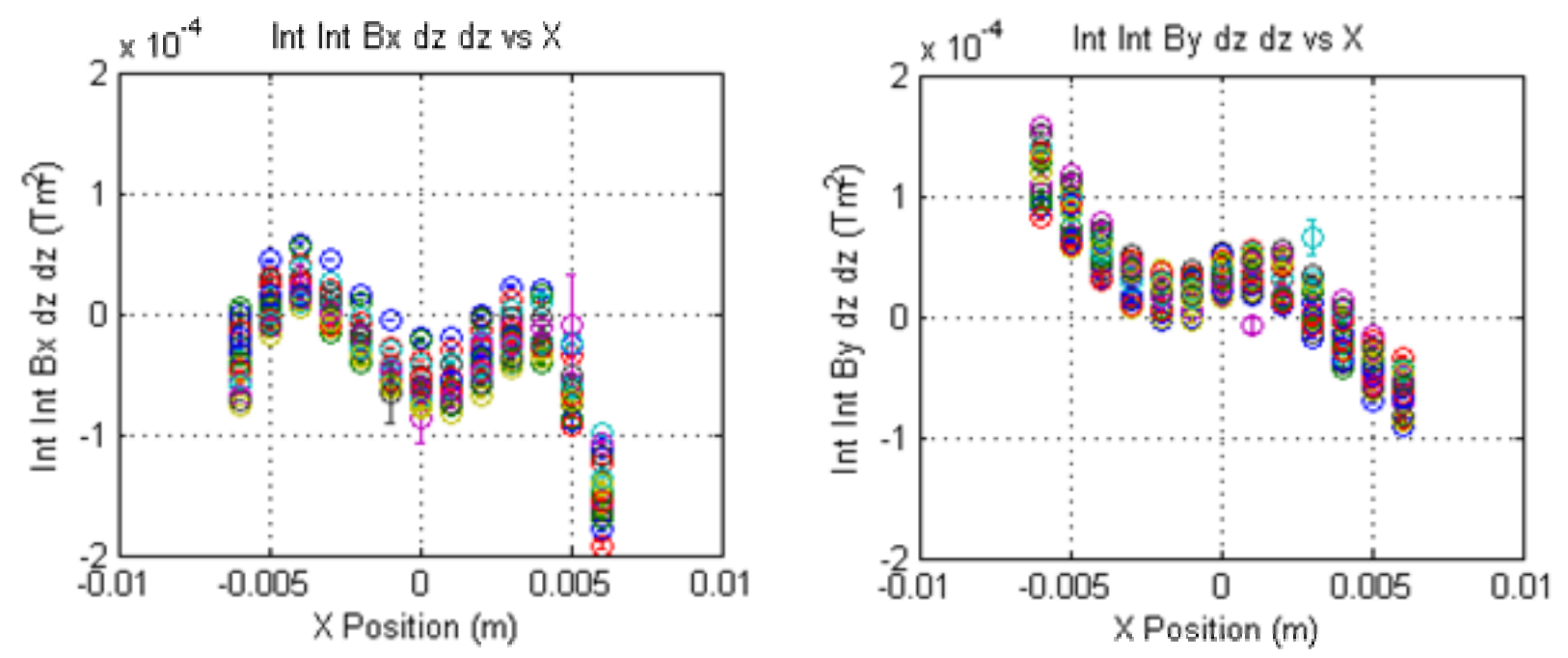

\begin{tabular}{|c|c|}
\hline O & 02-May-2007 \\
\hline 0 & $25-J$ \\
\hline 0 & $27-S$ \\
\hline 0 & 13-Nov-2007 \\
\hline 0 & $10-[$ \\
\hline 0 & 17-Jar \\
\hline 0 & $28-F$ \\
\hline 0 & 03-Apr \\
\hline 0 & 30-Ma \\
\hline 0 & $09-5$ \\
\hline 0 & 21-Aug \\
\hline 0 & $18-\mathrm{Se}$ \\
\hline 0 & $16-$ Oct-2008 \\
\hline 0 & $29-0 c t-2008$ \\
\hline 0 & $14-\mathrm{Nc}$ \\
\hline 0 & 08-Jan-2009 \\
\hline 0 & 17-Feb-2009 \\
\hline 0 & 19-Mar-2009 \\
\hline 0 & 15-Apr-2009 \\
\hline 0 & 29-May-2009 \\
\hline 0 & 06-Jul-2009 \\
\hline
\end{tabular}



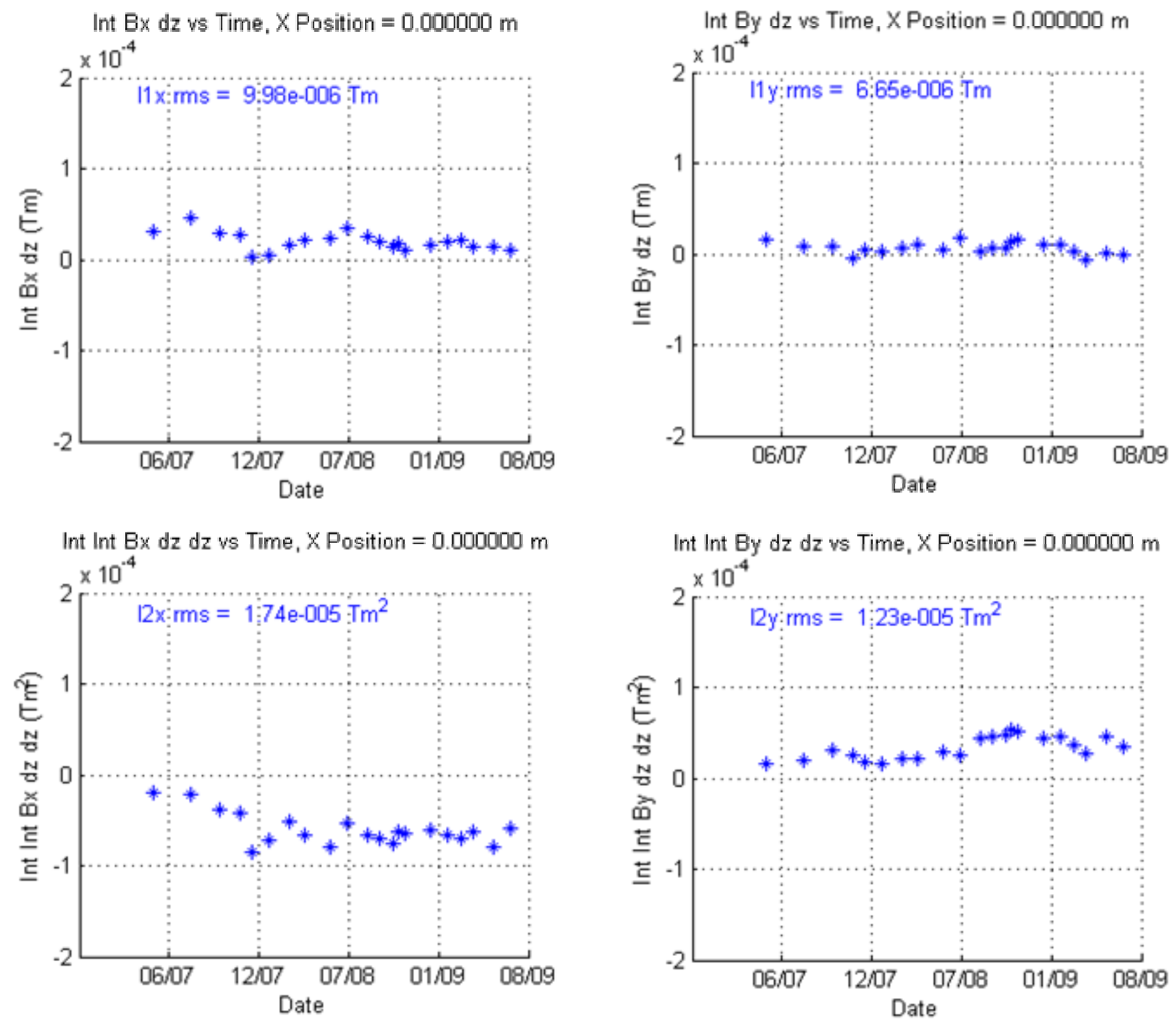

Figure 13 


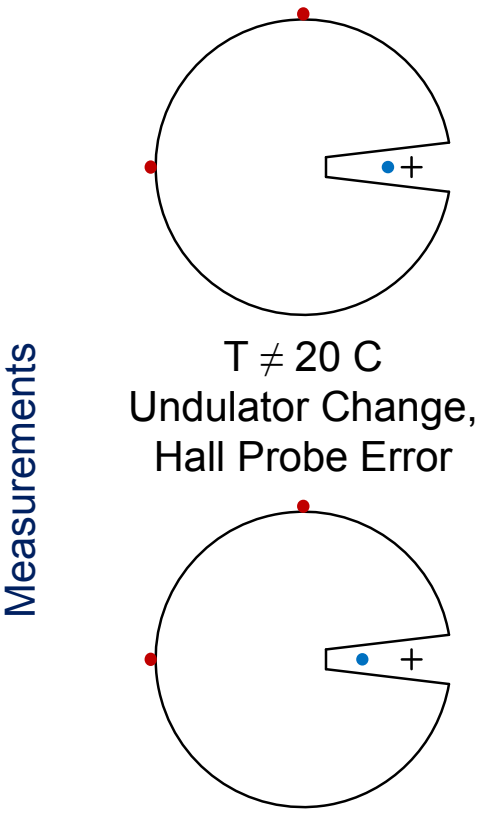

Hall Calibration Error, Probe Rotation Error

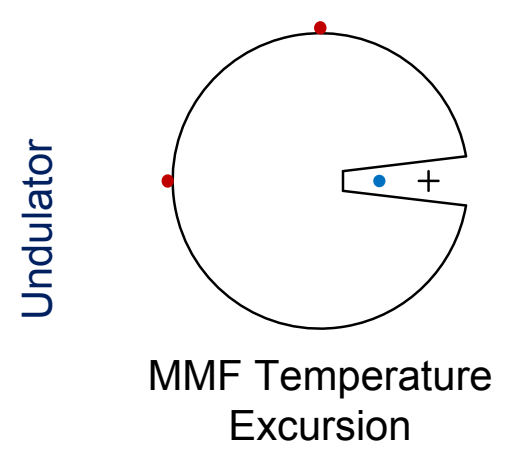

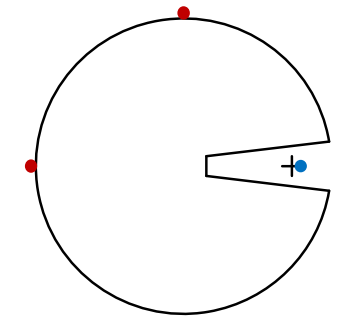

$\mathrm{K}_{\text {set }} \neq \mathrm{K}_{\text {ideal }}$

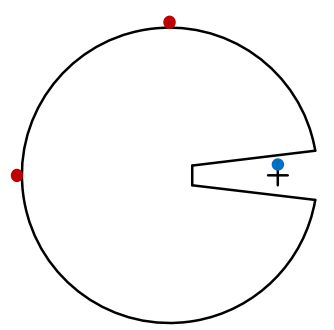

$\mathrm{B}_{\mathrm{x}} \mathrm{B}_{\mathrm{y}}$ Probe Positions

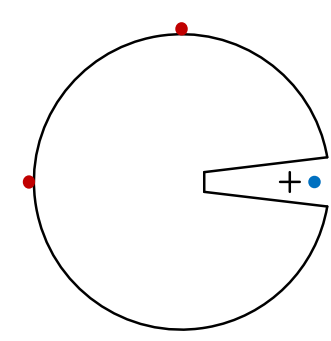

Undulator Bolts

Stretching

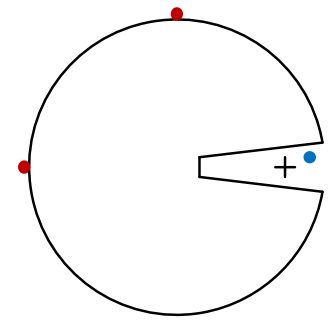

Fiducialization Error

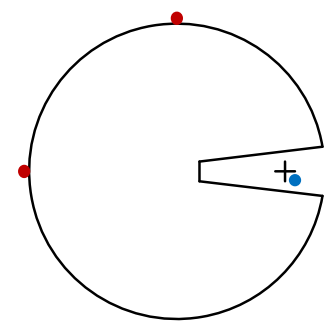

Planar Hall Effect

Figure 14 

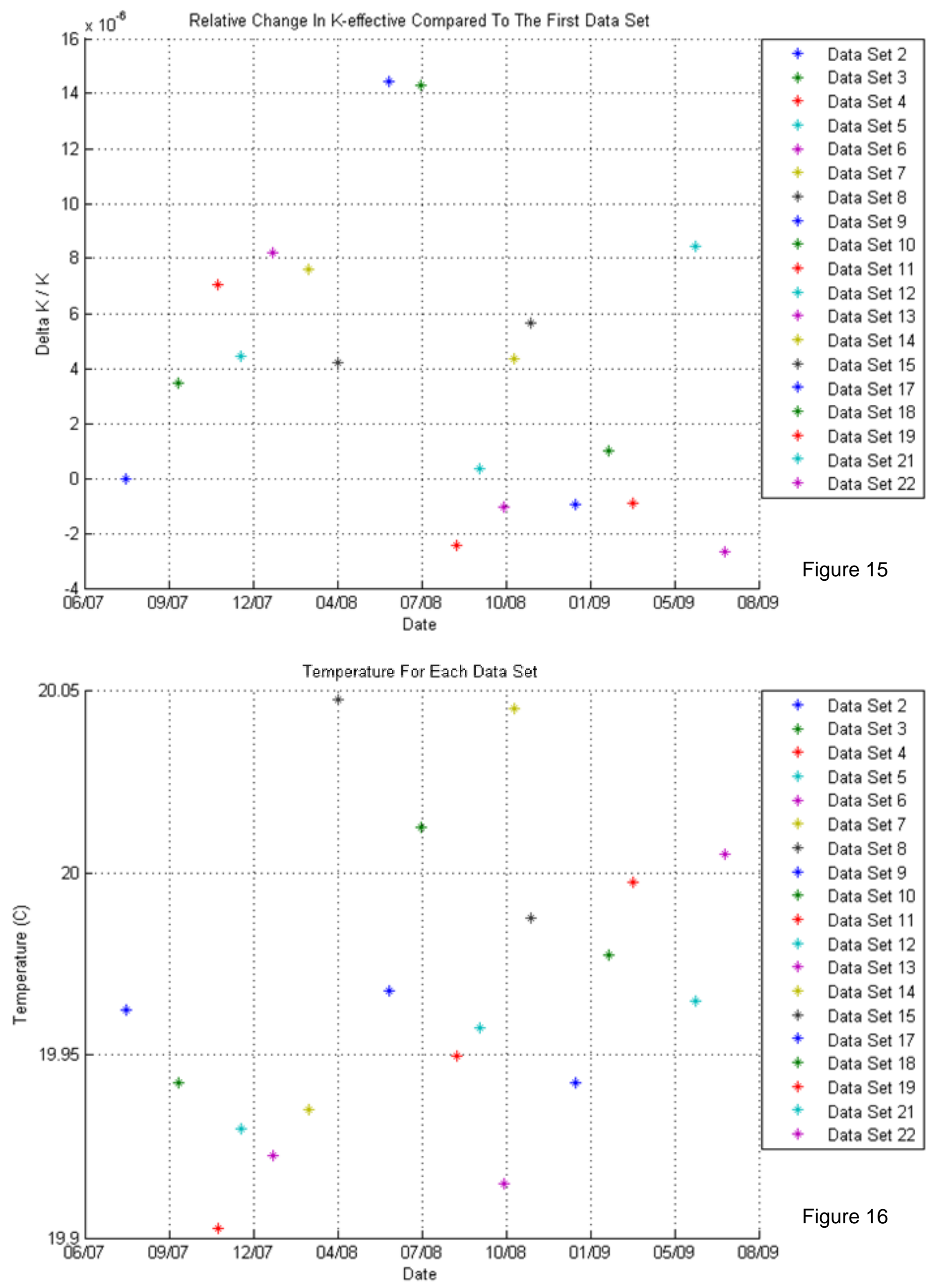
Change In Average Of The Tooling Ball X Positions

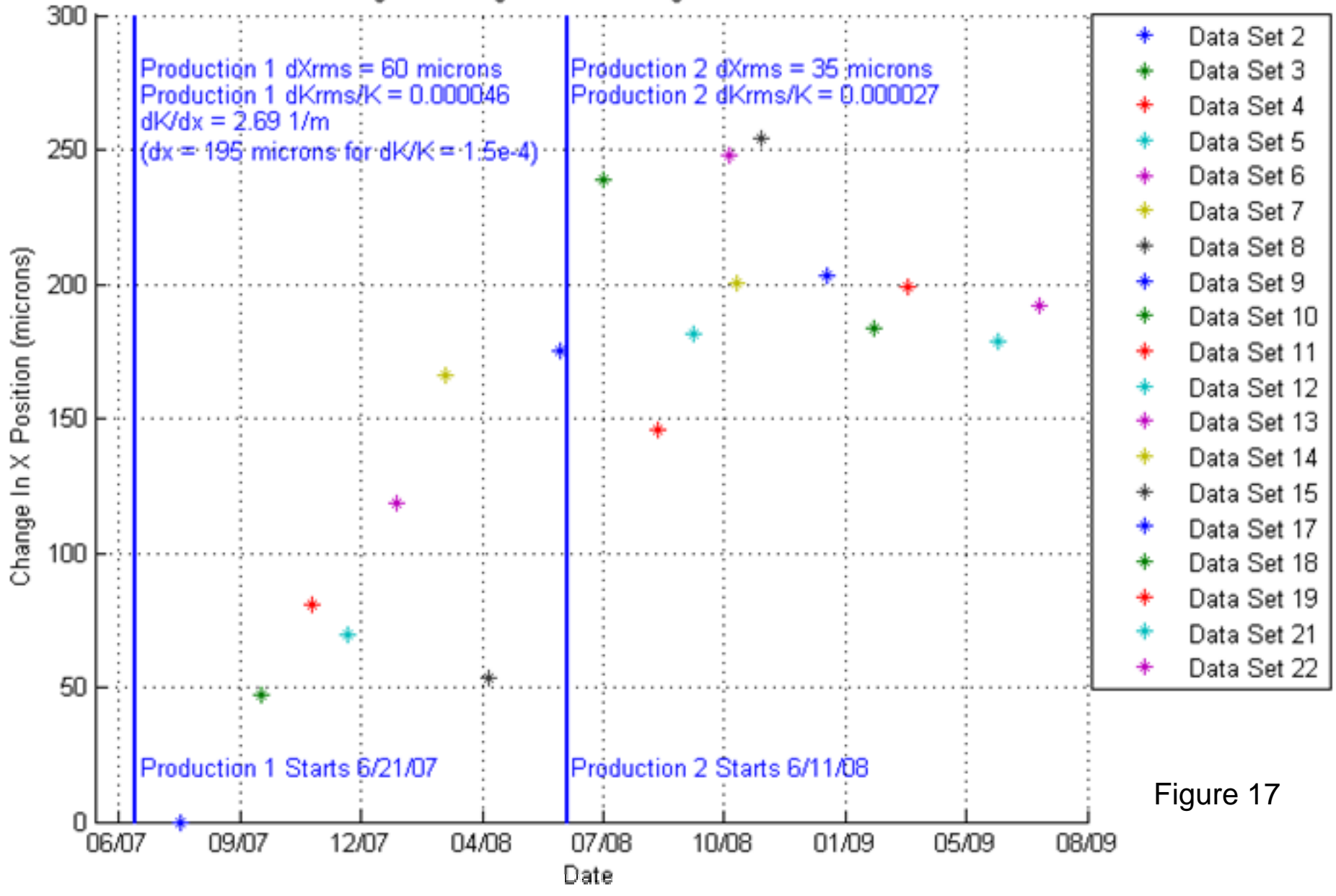

Change In Average Of The Tooling Ball Y Positions

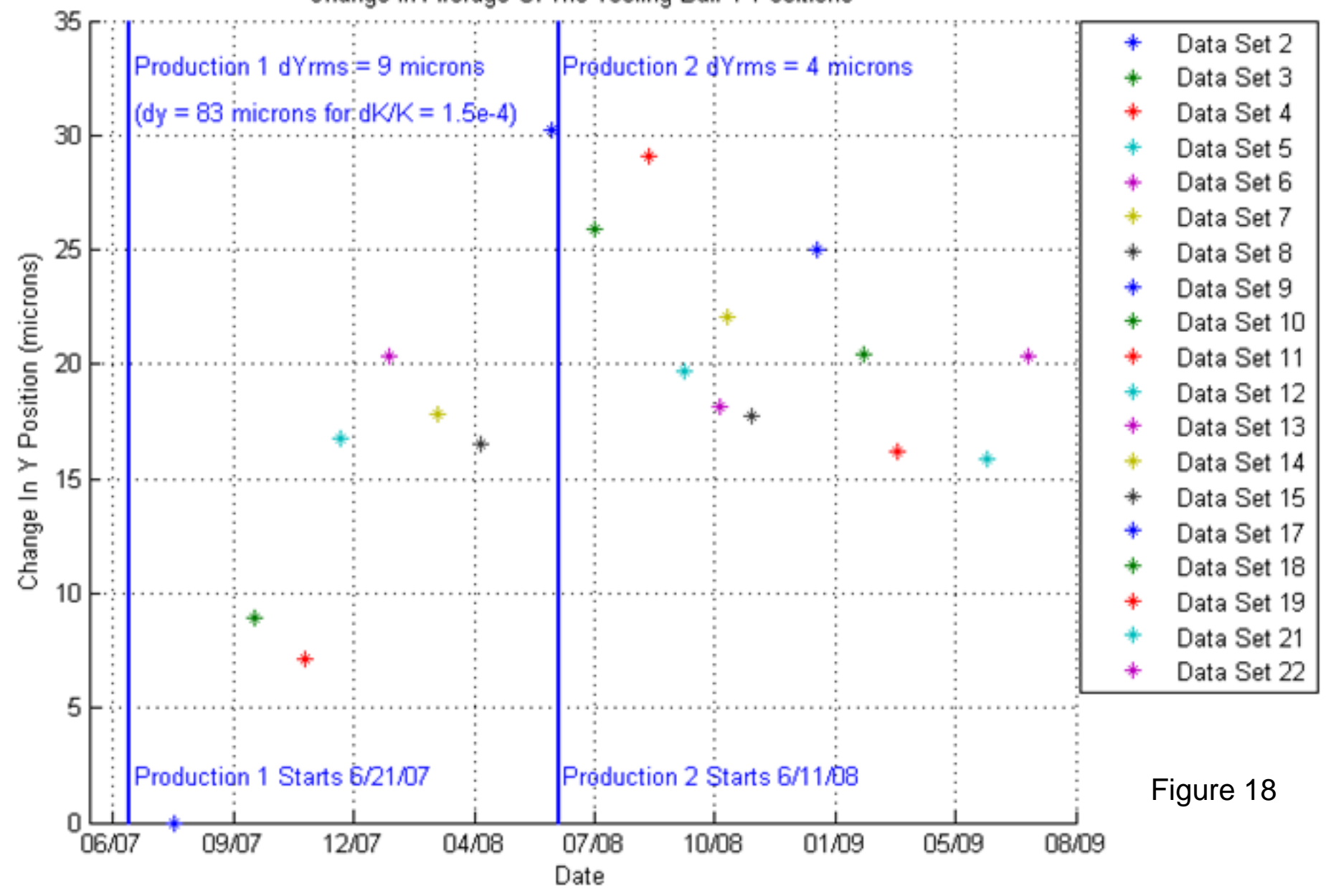




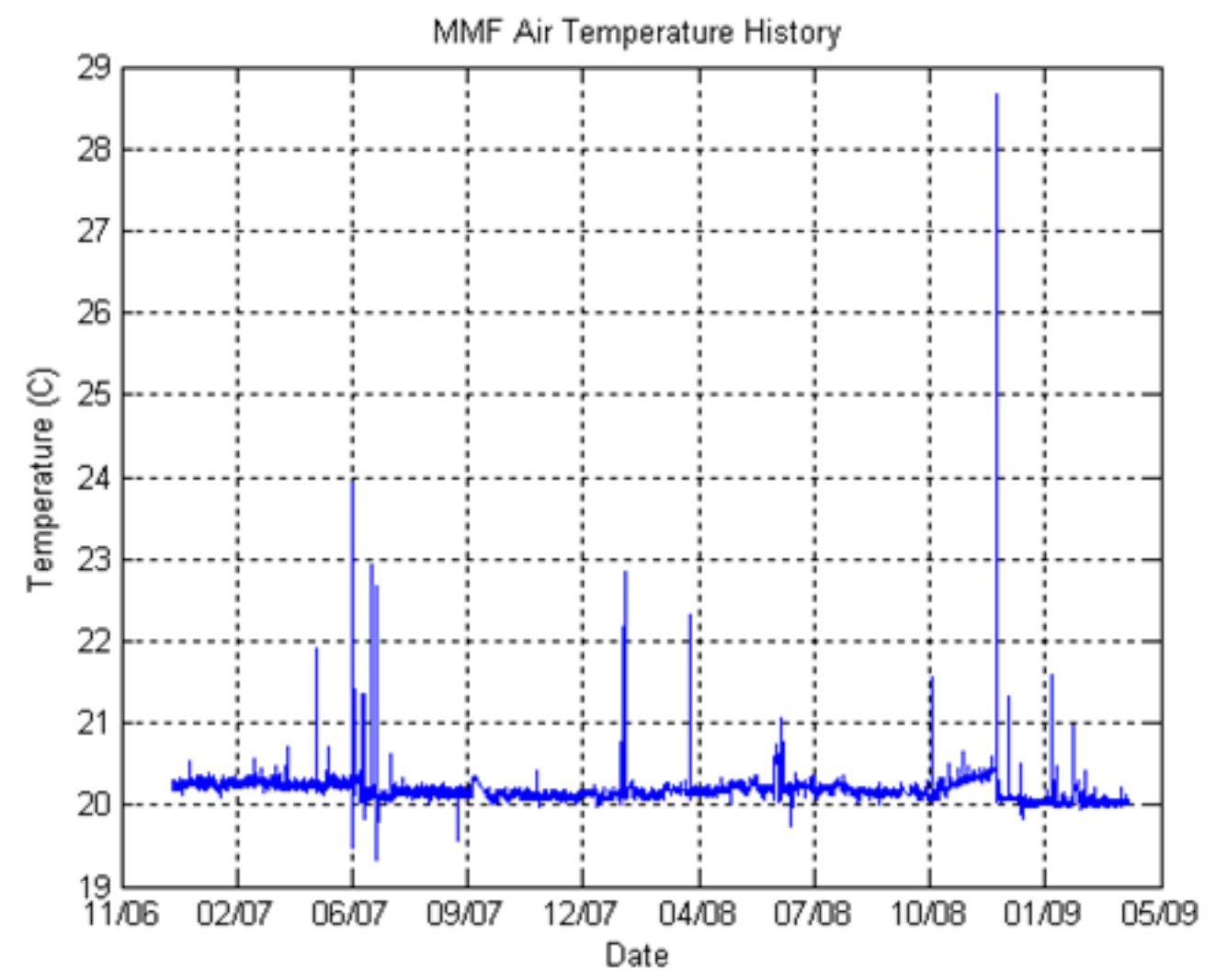

Figure 19

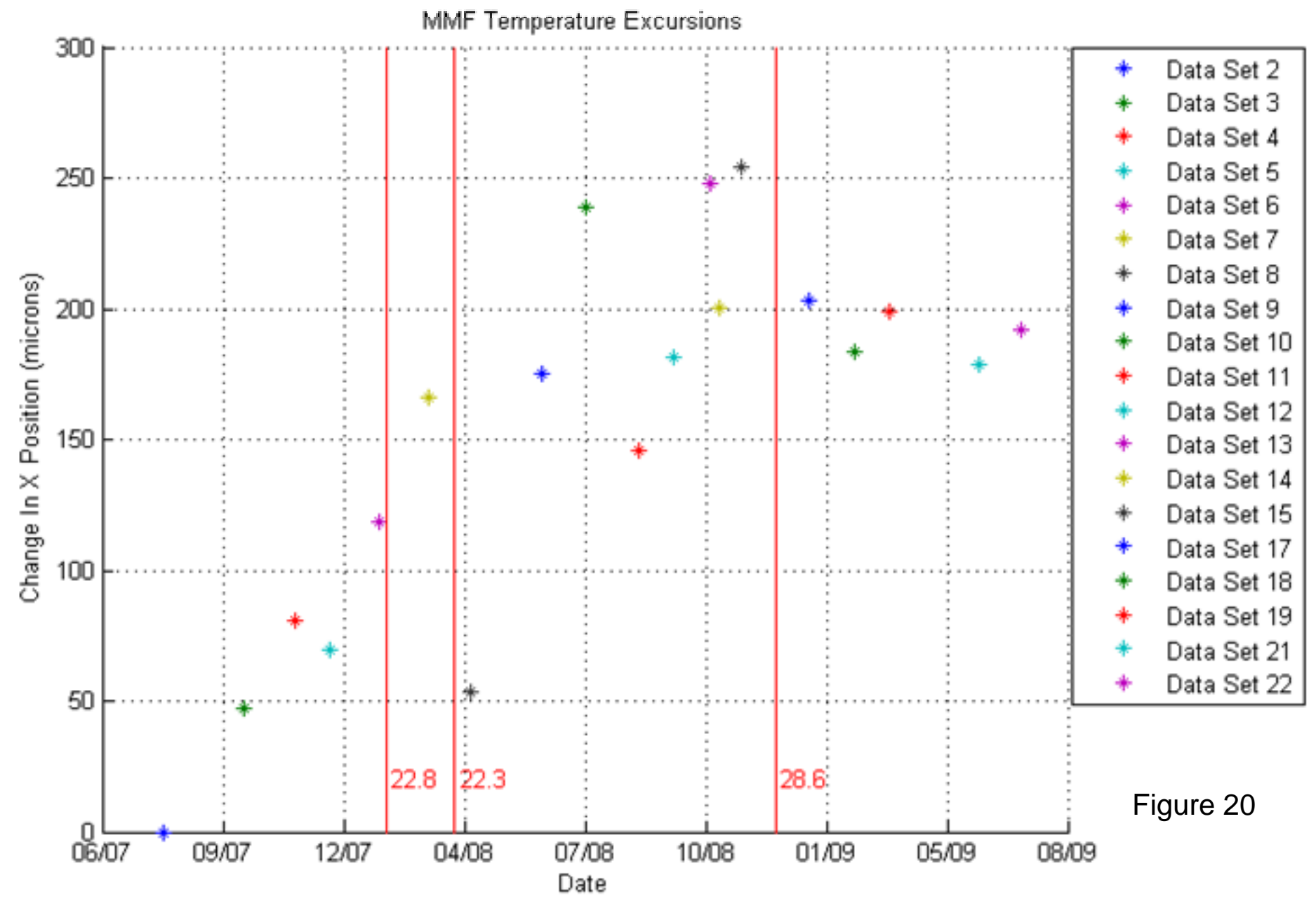



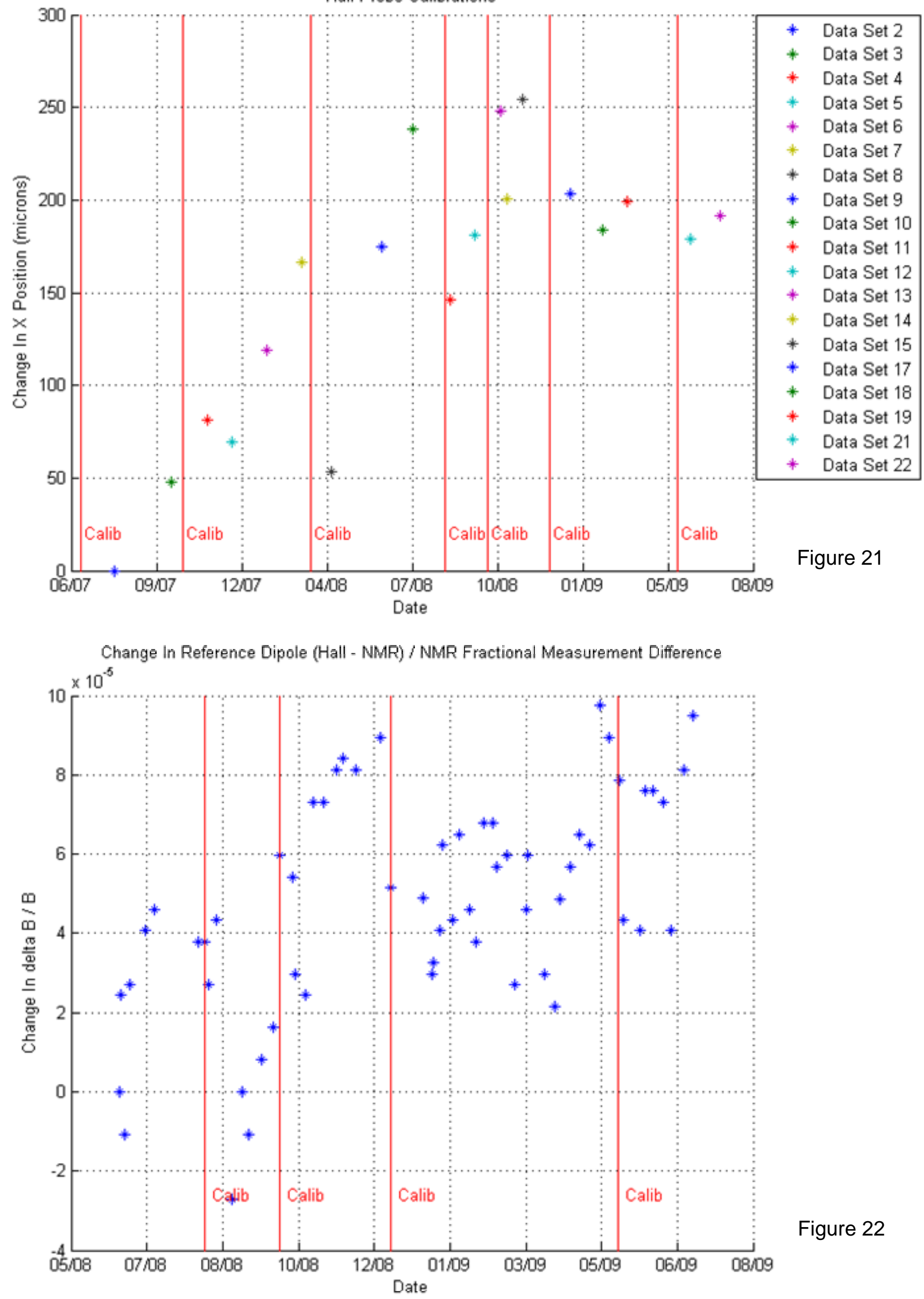

Figure 22 